Journal of Mathematics and Informatics

Vol. 18, 2020, 121-132

ISSN: 2349-0632 (P), 2349-0640 (online)

Published 14 March 2020

www. researchmathsci.org

DOI: http://dx.doi.org/10.22457/jmi.v18a10168

Journal of

Mathematics and

Informatics

\title{
On Positive Solution for a Class of Singular Elliptic System involving Critical Coupling Terms
}

\author{
Jie Yang ${ }^{1}$ and Zhi-Ying Deng ${ }^{2}$
}

${ }^{1}$ School of Science, Chongqing University of Posts and Telecommunications, Chongqing - 400065, Chongqing, China. E-mail: yangjie equ@qq.com

${ }^{2}$ Key Lab of Intelligent Analysis and Decision on Complex Systems, Chongqing University of Posts and Telecommunications, Chongqing - 400065, Chongqing, China. E-mail: dengzy@ cqupt.edu.cn

Received 22 February 2020; accepted 11 March 2020

Abstract. This paper deals with a class of singular semilinear elliptic system involving Hardy terms, critical coupling terms and negative-exponent terms. By using Ekeland variational principle and the Nehari set, we prove the existence of positive ground state weak solution.

Keywords: Semilinear elliptic system; Hardy term; Critical Sobolev exponents; The Ekeland variational principle

AMS Mathematics Subject Classification (2010): 35B33, 35J48, 35J50

\section{Introduction}

The aim of this paper is to establish the existence of nontrivial weak solution to the following semilinear elliptic system

$$
\begin{cases}-\Delta u-\mu \frac{u}{|x|^{2}}=\frac{\lambda \alpha_{1}}{\alpha_{1}+\beta_{1}} u^{\alpha_{1}-1} v^{\beta_{1}}+\frac{\alpha_{2}}{\alpha_{2}+\beta_{2}} f(x) u^{\alpha_{2}-1} v^{\beta_{2}}, & x \in \Omega, \\ -\Delta v-\mu \frac{v}{|x|^{2}}=\frac{\lambda \beta_{1}}{\alpha_{1}+\beta_{1}} u^{\alpha_{1}} v^{\beta_{1}-1}+\frac{\beta_{2}}{\alpha_{2}+\beta_{2}} f(x) u^{\alpha_{2}} v^{\beta_{2}-1}, & x \in \Omega, \\ u, v>0, & x \in \Omega, \\ u=v=0, & x \in \partial \Omega,\end{cases}
$$

where $\Omega \subset \mathbb{R}^{N}(N \geq 3)$ is a smooth bounded domain, $0 \in \Omega, \Delta$ is the Laplacian operator, $0 \leq \mu<\bar{\mu}, \bar{\mu} \triangleq\left(\frac{N-2}{2}\right)^{2}$ is the best Hardy constant, $\lambda>0$ is a real parameter, $\alpha_{1}, \beta_{1}>1, \alpha_{1}+\beta_{1}=2^{*}, 2^{*} \triangleq \frac{2 N}{N-2}$ is the critical Sobolev exponent, $\alpha_{2}, \beta_{2}>0, \alpha_{2}+\beta_{2}<2$ and $f: \Omega \rightarrow \mathbb{R}$ is a given non-negative nontrivial function. 


\section{Jie Yang and Zhi-Ying Deng}

By setting $\alpha_{2}, \beta_{2}$ larger than 0 rather 1 and $\alpha_{2}+\beta_{2}<2$, we find that at least one of the exponents of $u$ and $v$ is negative, namely $\min \left\{\alpha_{2}-1, \beta_{2}-1\right\}<0$. Hence problem (1) contains both negative exponent terms and critical Sobolev exponent terms.

Set $u=v, \alpha_{1}=\beta_{1}=\frac{2^{*}}{2}, \alpha_{2}=\beta_{2}>0, \alpha_{2}+\beta_{2}<1$, then system (1) reduces to the following equation

$$
\begin{cases}-\Delta u-\mu \frac{u}{|x|^{2}}=\lambda u^{2^{*}-1}+f(x) u^{q}, & x \in \Omega, \\ u>0, & x \in \Omega, \\ u=0, & x \in \partial \Omega,\end{cases}
$$

where $0<q<1$. The existence of solution for problems like (2) are studied extensively and many results have been found [1-3].

In recent years, much attention has been paid to critical elliptic systems, but the exponents in these systems are usually positive (see [4-7]). Hsu and Li [8] studied the following semilinear elliptic system:

$$
\begin{cases}-\Delta u-\mu \frac{u}{|x|^{2}}=\lambda|u|^{q-2} u+\frac{2 \alpha}{\alpha+\beta} \frac{|u|^{\alpha-2} u|v|^{\beta}}{|x|^{s}}, & \text { in } \Omega \backslash\{0\}, \\ -\Delta v-\mu \frac{v}{|x|^{2}}=\sigma|v|^{q-2} v+\frac{2 \beta}{\alpha+\beta} \frac{|u|^{\alpha}|v|^{\beta-2} v}{|x|^{s}}, & \text { in } \Omega \backslash\{0\}, \\ u=v=0, & \text { on } \partial \Omega,\end{cases}
$$

where $\lambda, \sigma>0,0 \leq \mu<\bar{\mu}=\left(\frac{N-2}{2}\right)^{2}, 1 \leq q<2,0 \leq s<2$, and $\alpha, \beta>1$ satisfy $\alpha+\beta=2^{*}(s)=\frac{2(N-s)}{N-2}$. They obtained the multiplicity of positive solutions by variational methods when the parameters $\lambda, \sigma$ satisfy $0<\lambda^{\frac{2}{2-q}}+\sigma^{\frac{2}{2-q}}<\Lambda$, where $\Lambda$ is a certain positive constant.

The existence and nonexistence of solutions of elliptic systems with negative exponents are also studied in [9-11] and the papers therein. However, to the best of our knowledge, there are few results for the elliptic system (1) involving both critical Sobolev exponents and negative exponents. Therefore, it is meaningful for us to study the elliptic systems (1).

However, the first difficulty to study the existence of solutions of (1) is that the functional corresponding with (1) is no longer Frechet differentiable. Therefore, there exists no $(P S)_{c}$ sequence for the functional. The second difficulty is due to the lack of 
On Positive Solution for a Class of Singular Elliptic System involving Critical Coupling Terms

compactness of the embedding $W_{0}^{1,2}(\Omega) \subseteq L^{2^{*}}(\Omega)$. By using the Ekeland variational principle and the Vitali theorem, we overcome the above difficulties and obtain some new results.

The main result of this paper is the following theorem.

Theorem 1.1. Suppose $f \in L^{\infty}(\Omega)$ and $\Omega \subset \mathbb{R}^{N}$ is bounded, $0 \in \Omega$, then there exists constants $\lambda^{*}>0$ and $\bar{C}$ such that for all $\lambda \in\left(0, \lambda^{*}\right)$ and $|f|_{\infty}<\bar{C}$, problem (1) has a positive ground state weak solution.

\section{Preliminaries and some technical lemmas}

Throughout this paper, the space $W_{0}^{1,2}(\Omega)$ represents the completion of $C_{0}^{\infty}(\Omega)$ with the $\operatorname{norm}\left(\int_{\Omega}|\nabla \cdot|^{2} \mathrm{~d} x\right)^{\frac{1}{2}}$. Problem (1) is related to the well-known Hardy inequality

$$
\int_{\Omega} \frac{|u|^{2}}{|x|^{2}} \mathrm{~d} x \leq \frac{1}{\bar{\mu}} \int_{\Omega}|\nabla u|^{2} \mathrm{~d} x, \quad \forall u \in W_{0}^{1,2}(\Omega) .
$$

In space $W_{0}^{1,2}(\Omega)$, we employ the following norm

$$
\|u\|_{\mu}=\left[\int_{\Omega}\left(|\nabla u|^{2}-\mu \frac{|u|^{2}}{|x|^{2}}\right) \mathrm{d} x\right]^{\frac{1}{2}}, \quad \forall \mu \in[0, \bar{\mu}) .
$$

By Hardy inequality, the above norm is equivalent to the usual norm $\left(\int_{\Omega}|\nabla \cdot|^{2} \mathrm{~d} x\right)^{\frac{1}{2}}$ in $W_{0}^{1,2}(\Omega)$. In this paper, we work on the product space $E=W_{0}^{1,2}(\Omega) \times W_{0}^{1,2}(\Omega)$ with the norm

$$
\|(u, v)\|=\left(\|u\|_{\mu}^{2}+\|v\|_{\mu}^{2}\right)^{\frac{1}{2}} .
$$

Besides, the space $L^{p}(\Omega), 1 \leq p<\infty$ denotes the usual Lebesgue space.

For simplicity, we write $q=\alpha_{2}+\beta_{2}$. A pair of functions $(u, v) \in E$ is said to be a weak solution of problem (1) if

$$
\begin{aligned}
& \int_{\Omega}\left(\nabla u \nabla \varphi_{1}+\nabla v \nabla \varphi_{2}-\mu \frac{u \varphi_{1}}{|x|^{2}}-\mu \frac{v \varphi_{2}}{|x|^{2}}\right) \mathrm{d} x-\frac{\lambda}{2^{*}} \int_{\Omega}\left(\alpha_{1} u^{\alpha_{1}-1} v^{\beta_{1}} \varphi_{1}+\beta_{1} u^{\alpha_{1}} v^{\beta_{1}-1} \varphi_{2}\right) \mathrm{d} x \\
& -\int_{\Omega} \frac{f(x)}{q}\left(\alpha_{2} u^{\alpha_{2}-1} v^{\beta_{2}} \varphi_{1}+\beta_{2} u^{\alpha_{2}} v^{\beta_{2}-1} \varphi_{2}\right) \mathrm{d} x=0, \quad \forall\left(\varphi_{1}, \varphi_{2}\right) \in E .
\end{aligned}
$$

The corresponding energy functional $I: E \rightarrow \mathbb{R}$ associated with the problem (1) is defined by 
Jie Yang and Zhi-Ying Deng

$$
\begin{aligned}
I(u, v)= & \frac{1}{2} \int_{\Omega}\left(|\nabla u|^{2}+|\nabla v|^{2}-\mu \frac{|u|^{2}}{|x|^{2}}-\mu \frac{|v|^{2}}{|x|^{2}}\right) \mathrm{d} x \\
& -\frac{\lambda}{2^{*}} \int_{\Omega}|u|^{\alpha_{1}}|v|^{\beta_{1}} \mathrm{~d} x-\frac{1}{q} \int_{\Omega} f(x)|u|^{\alpha_{2}}|v|^{\beta_{2}} \mathrm{~d} x .
\end{aligned}
$$

For any $\mu \in[0, \bar{\mu}), \alpha, \beta>1$ and $\alpha+\beta=2^{*}$, we define the following constants:

$$
\begin{gathered}
S=S(\mu) \triangleq \inf _{u \in W_{0}^{1,2}(\Omega) \backslash\{0\}} \frac{\int_{\Omega}\left(|\nabla u|^{2}-\mu \frac{|u|^{2}}{|x|^{2}}\right) \mathrm{d} x}{\left(\int_{\Omega}|u|^{2^{*}} \mathrm{~d} x\right)^{\frac{2}{2^{*}}}}, \\
S_{\alpha, \beta} \triangleq \inf _{u, v \in W_{0}^{1,2}(\Omega) \backslash\{0\}} \frac{\|\left.(u, v)\right|^{2}}{\left(\int_{\Omega}|u|^{\alpha}|v|^{\beta} \mathrm{d} x\right)^{\frac{2}{2^{*}}}}, \\
\bar{C} \triangleq \frac{2^{*}-2}{2^{*}-q}\left(\frac{2-q}{2^{*}-q}\right)^{\frac{2-q}{2^{*}-2}} S_{\alpha_{1}, \beta_{1}}^{\frac{2^{*}-q}{2^{*}-2}}|\Omega|^{\frac{q-2^{*}}{2^{*}}}\left[\left(\frac{\alpha_{2}}{q}\right)^{\frac{2}{2-q}}+\left(\frac{\beta_{2}}{q}\right)^{\frac{2}{2-q}}\right]^{\frac{q-2}{2}} .
\end{gathered}
$$

For each $(u, v) \in E$, we define $J(t)=I((t u, t v))$, namely

$$
\begin{gathered}
J(t)=\frac{t^{2}}{2}\|(u, v)\|^{2}-\lambda \frac{t^{2^{*}}}{2^{*}} \int_{\Omega}|u|^{\alpha_{1}}|v|^{\beta_{1}} \mathrm{~d} x-\frac{t^{q}}{q} \int_{\Omega} f(x)|u|^{\alpha_{2}}|v|^{\beta_{2}} \mathrm{~d} x, \\
J^{\prime}(t)=t\|(u, v)\|^{2}-\lambda t^{2^{*}-1} \int_{\Omega}|u|^{\alpha_{1}}|v|^{\beta_{1}} \mathrm{~d} x-t^{q-1} \int_{\Omega} f(x)|u|^{\alpha_{2}}|v|^{\beta_{2}} \mathrm{~d} x, \\
J^{\prime \prime}(t)=\|(u, v)\|^{2}-\lambda\left(2^{*}-1\right) t^{2^{*}-2} \int_{\Omega}|u|^{\alpha_{1}}|v|^{\beta_{1}} \mathrm{~d} x+(1-q) t^{q-2} \int_{\Omega} f(x)|u|^{\alpha_{2}}|v|^{\beta_{2}} \mathrm{~d} x .
\end{gathered}
$$

Meanwhile, we define Nehari set

$$
\begin{aligned}
\Lambda & =\left\{(u, v) \in E \backslash\{(0,0)\} \mid J^{\prime}(1)=0\right\} \\
& =\left\{\left.(u, v) \in E \backslash\{(0,0)\}\left|\|(u, v)\|^{2}-\lambda \int_{\Omega}\right| u\right|^{\alpha_{1}}|v|^{\beta_{1}} \mathrm{~d} x-\int_{\Omega} f(x)|u|^{\alpha_{2}}|v|^{\beta_{2}} \mathrm{~d} x=0\right\},
\end{aligned}
$$

which contains all solutions of problem (1). We make the following splitting for $\Lambda$ : $\Lambda^{ \pm}=\left\{(u, v) \in \Lambda \mid J^{\prime \prime}(1)>(<) 0\right\}, \Lambda^{0}=\left\{(u, v) \in \Lambda \mid J^{\prime \prime}(1)=0\right\}$, i.e.

$$
\begin{aligned}
& \Lambda^{+}=\left\{\left.(u, v) \in \Lambda\left|(2-q)\|(u, v)\|^{2}-\lambda\left(2^{*}-q\right) \int_{\Omega}\right| u\right|^{\alpha_{1}}|v|^{\beta_{1}} \mathrm{~d} x>0\right\}, \\
& \Lambda^{0}=\left\{\left.(u, v) \in \Lambda\left|(2-q)\|(u, v)\|^{2}-\lambda\left(2^{*}-q\right) \int_{\Omega}\right| u\right|^{\alpha_{1}}|v|^{\beta_{1}} \mathrm{~d} x=0\right\}, \\
& \Lambda^{-}=\left\{\left.(u, v) \in \Lambda\left|(2-q)\|(u, v)\|^{2}-\lambda\left(2^{*}-q\right) \int_{\Omega}\right| u\right|^{\alpha_{1}}|v|^{\beta_{1}} \mathrm{~d} x<0\right\} .
\end{aligned}
$$

In the following, some technical lemmas will be elaborated as follows.

Lemma 2.1. The energy functional $I(u, v)$ is coercive and bounded below on $\Lambda$. 
On Positive Solution for a Class of Singular Elliptic System involving Critical Coupling Terms

Proof: By the Young inequality, $\alpha_{2}, \beta_{2}<q$ and the Holder inequality, we have

$$
\begin{aligned}
& \int_{\Omega} f(x)|u|^{\alpha_{2}}|v|^{\beta_{2}} \mathrm{~d} x \leq|f|_{\infty}\left(\frac{\alpha_{2}}{q}|u|_{q}^{q}+\frac{\beta_{2}}{q}|v|_{q}^{q}\right) \\
\leq & |f|_{\infty}|\Omega|^{\frac{2^{*}-q}{2^{*}}} S^{-\frac{q}{2}}\left(\left(\frac{\alpha_{2}}{q}\right)^{\frac{2}{2-q}}+\left(\frac{\beta_{2}}{q}\right)^{\frac{2}{2-q}}\right)^{\frac{2-q}{2}}\|(u, v)\|^{q} \triangleq C_{1}\|(u, v)\|^{q},
\end{aligned}
$$

where $|\Omega|$ is the Lebesgue measure of $\Omega$. Based on above inequality, we have

$$
\begin{aligned}
I(u, v) & =\left(\frac{1}{2}-\frac{1}{2^{*}}\right)\|(u, v)\|^{2}-\left(\frac{1}{q}-\frac{1}{2^{*}}\right) \int_{\Omega} f(x)|u|^{\alpha_{2}}|v|^{\beta_{2}} \mathrm{~d} x \\
& \geq\left(\frac{1}{2}-\frac{1}{2^{*}}\right)\|(u, v)\|^{2}-C_{1}\left(\frac{1}{q}-\frac{1}{2^{*}}\right)\|(u, v)\|^{q}, \quad \forall(u, v) \in \Lambda .
\end{aligned}
$$

Since $0<q<2$, this implies that the conclusion is true.

Lemma 2.2. There exists a constant $\lambda_{1}>0$, such that $\Lambda^{0}=\varnothing$ for all $\lambda<\lambda_{1}$.

Proof: To the contrary, we suppose that there exists $(\bar{u}, \bar{v}) \in \Lambda^{0} \subset \Lambda$, which means

$$
(2-q)\|(\bar{u}, \bar{v})\|^{2}-\lambda\left(2^{*}-q\right) \int_{\Omega}|\bar{u}|^{\alpha_{1}}|\bar{v}|^{\beta_{1}} \mathrm{~d} x=0 .
$$

Based on the definition of $\Lambda$, it is easy to find that $\bar{u}, \bar{v} \not \equiv 0$. From (4) and Sobolev inequality it follows that

Consequently,

$$
\frac{\|(\bar{u}, \bar{v})\|^{2}}{\int_{\Omega}|\bar{u}|^{\alpha_{1}}|\bar{v}|^{\beta_{1}} \mathrm{~d} x}=\frac{\lambda\left(2^{*}-q\right)}{2-q} \geq \frac{\|(\bar{u}, \bar{v})\|^{2}}{S_{\alpha_{1}, \beta_{1}}^{-\frac{2^{*}}{2}}\|(\bar{u}, \bar{v})\|^{2^{*}}} .
$$

$$
\|(\bar{u}, \bar{v})\| \geq\left[\frac{S_{\alpha_{1}, \beta_{1}}^{\frac{2^{*}}{2}}(2-q)}{\lambda\left(2^{*}-q\right)}\right]^{\frac{1}{2^{*}-2}} .
$$

On the other hand, from $(\bar{u}, \bar{v}) \in \Lambda$, we have

$$
\lambda \int_{\Omega}|\bar{u}|^{\alpha_{1}}|\bar{v}|^{\beta_{1}} \mathrm{~d} x=\|(\bar{u}, \bar{v})\|^{2}-\int_{\Omega} f(x)|\bar{u}|^{\alpha_{2}}|\bar{v}|^{\beta_{2}} \mathrm{~d} x,
$$

combining with (4) and (3), we have

Consequently,

$$
\frac{2^{*}-2}{2^{*}-q}\|(\bar{u}, \bar{v})\|^{2}-C_{1}\|(\bar{u}, \bar{v})\|^{q} \leq 0 .
$$


Jie Yang and Zhi-Ying Deng

$$
\|(\bar{u}, \bar{v})\| \leq\left(\frac{C_{1}\left(2^{*}-q\right)}{2^{*}-2}\right)^{\frac{1}{2-q}} .
$$

Let

$$
\lambda_{1}=S_{\alpha_{1}, \beta_{1}}^{\frac{2^{*}}{2}} \frac{2-q}{2^{*}-q}\left(\frac{2^{*}-2}{C_{1}\left(2^{*}-q\right)}\right)^{\frac{2^{*}-2}{2-q}},
$$

then, by (5) and (6), we have $\lambda>\lambda_{1}$ which is a contradiction, thus $\Lambda^{0}=\varnothing$.

Lemma 2.3. There exists a positive number $\lambda_{2}>0$ such that if $\lambda<\lambda_{2}$,

(i) $I(u, v)<0$ for any $(u, v) \in \Lambda^{+}$;

(ii) $I(u, v)>0$ for any $(u, v) \in \Lambda^{-}$.

Proof: First, we prove (i). Arguing by contradiction, we assume that there exists $(u, v) \in \Lambda^{+}$which satisfies $I(u, v) \geq 0$, i.e.

$$
\frac{1}{2}\|(u, v)\|^{2}-\frac{\lambda}{2^{*}} \int_{\Omega}|u|^{\alpha_{1}}|v|^{\beta_{1}} \mathrm{~d} x-\frac{1}{q} \int_{\Omega} f(x)|u|^{\alpha_{2}}|v|^{\beta_{2}} \mathrm{~d} x \geq 0 .
$$

From $(u, v) \in \Lambda$, it follows that

$$
\|(u, v)\| \geq\left[S_{\alpha_{1}, \beta_{1}}^{\frac{2^{*}}{2}} \frac{2^{*}(2-q)}{2 \lambda\left(2^{*}-q\right)}\right]^{\frac{1}{2^{*}-2}} .
$$

On the other hand, from $(u, v) \in \Lambda^{+} \subset \Lambda$, we have

$$
\left(2^{*}-2\right)\|(u, v)\|^{2}-\left(2^{*}-q\right) \int_{\Omega} f(x)|u|^{\alpha_{2}}|v|^{\beta_{2}} \mathrm{~d} x<0,
$$

combining with (3), we have

$$
\|(u, v)\|<\left(\frac{2^{*}-q}{2^{*}-2} C_{1}\right)^{\frac{1}{2-q}}
$$

Let

$$
\bar{\lambda}_{2}=S_{\alpha_{1}, \beta_{1}}^{-\frac{2^{*}}{2}} \frac{2\left(2^{*}-q\right)}{2^{*}(2-q)}\left(\frac{2^{*}-q}{2^{*}-2} C_{1}\right)^{\frac{2^{*}-2}{2-q}} .
$$

Then, by (7) and (8), we have $\lambda>\bar{\lambda}_{2}$ which is a contradiction, thus $I(u, v)<0$.

In the following, we prove (ii). Let

$$
\tilde{\lambda}_{2}=S_{\alpha_{1}, \beta_{1}}^{\frac{2^{*}}{2}} \frac{2-q}{2^{*}-q}\left[\frac{q\left(2^{*}-2\right)}{2\left(2^{*}-q\right) C_{1}}\right]^{\frac{2^{*}-2}{2-q}},
$$

then by the same method, it can be proved that when $\lambda<\tilde{\lambda}_{2}$, we have $I(u, v)>0$ for any $(u, v) \in \Lambda^{-}$. By taking $\lambda_{2}=\min \left\{\bar{\lambda}_{2}, \tilde{\lambda}_{2}\right\}$, the conclusion is proved. 
On Positive Solution for a Class of Singular Elliptic System involving Critical Coupling Terms

Lemma 2.4. If $|f|_{\infty}<\bar{C}$, there exists a positive number $\lambda_{3} \in(0,1)$ such that $\Lambda^{+}$is nonempty and closed for any $\lambda<\lambda_{3}$.

Proof: Let

$$
\phi(t)=t^{2-q}\|(u, v)\|^{2}-\lambda t^{2^{*}-q} \int_{\Omega}|u|^{\alpha_{1}}|v|^{\beta_{1}} \mathrm{~d} x, \quad \forall(u, v) \in E \backslash\{(0,0)\},
$$

and $t_{\max }>0$ satisfy $\phi^{\prime}\left(t_{\max }\right)=0$. By calculation we find

$$
t_{\max }=\left[\frac{(2-q)\|(u, v)\|^{2}}{\lambda\left(2^{*}-q\right) \int_{\Omega}|u|^{\alpha_{1}}|v|^{\beta_{1}} \mathrm{~d} x}\right]^{\frac{1}{2^{*}-2}} .
$$

From $2^{*}>2$, it follows that $\phi(t)$ increases monotonically on $\left(0, t_{\max }\right)$ and decreases monotonically on $\left(t_{\max },+\infty\right)$. So $\phi(t)$ gets its maximum at $t_{\max }$ and

$$
\phi\left(t_{\max }\right)=\frac{2^{*}-2}{2^{*}-q}\left[\frac{2-q}{\lambda\left(2^{*}-q\right)}\right]^{\frac{2-q}{2^{*}-2}}\left[\frac{\|(u, v)\|^{\left(22^{*}-q\right)}}{\left(\int_{\Omega}|u|^{\alpha_{1}}|v|^{\beta_{1}} \mathrm{~d} x\right)^{2-q}}\right]^{\frac{1}{2^{*}-2}} .
$$

If $\lambda<1$, combining with $|f|_{\infty}<\bar{C}$ and (9), we have

$$
0<\int_{\Omega} f(x)|u|^{\alpha_{1}}|v|^{\beta_{1}} \mathrm{~d} x<\phi\left(t_{\max }\right) .
$$

From $\phi(t)$ increases monotonically on $\left(0, t_{\max }\right)$, we know there exists only one $t^{+}=t_{u, v}^{+}<t_{\max }$ such that

$$
\phi\left(t^{+}\right)=\int_{\Omega} f(x)|u|^{\alpha_{1}}|v|^{\beta_{1}} \mathrm{~d} x \text { and } \phi^{\prime}\left(t^{+}\right)>0,
$$

which implies $t^{+} u \in \Lambda^{+}$. Therefore $\Lambda^{+} \neq \varnothing$.

Lemma 2.5. If $(u, v) \in \Lambda^{+}$, then there exists a positive number $\epsilon>0$ and a differentiable function $h=h(t)>0$, where $t<\epsilon$ such that $h(0)=1$ and $h(t)\left(u+t \varphi_{1}, v+t \varphi_{2}\right) \in \Lambda^{+}$ for all $\left(\varphi_{1}, \varphi_{2}\right) \in E$.

Proof: By applying the method in [12,13], we define $H: \mathbb{R} \times E \rightarrow \mathbb{R}$ by

$$
\begin{aligned}
H\left(t, \varphi_{1}, \varphi_{2}\right)= & t^{2-q}\left\|\left(u+\varphi_{1}, v+\varphi_{2}\right)\right\|^{2}-\int_{\Omega} f(x)\left|u+\varphi_{1}\right|^{\alpha_{2}}\left|v+\varphi_{2}\right|^{\beta_{2}} \mathrm{~d} x \\
& -\lambda t^{2^{*}-q} \int_{\Omega}\left|u+\varphi_{1}\right|^{\alpha_{1}}\left|v+\varphi_{2}\right|^{\beta_{1}} \mathrm{~d} x .
\end{aligned}
$$

Since $u \in \Lambda^{+} \subset \Lambda$, we have $H(1,0,0)=0$ and

$$
H_{t}(1,0,0)=(2-q)\|(u, v)\|^{2}-\lambda\left(2^{*}-q\right) \int_{\Omega} \lambda|u|^{\alpha_{1}}|v|^{\beta_{1}} \mathrm{~d} x>0 .
$$

According to the implicit function theorem at the point $(1,0,0)$, there exist an $\bar{\epsilon}>0$ and a continuous function $h=h(t)>0$, where $t<\bar{\epsilon}$, such that 
Jie Yang and Zhi-Ying Deng

$$
h(0)=1, \quad h(t)\left(u+t \varphi_{1}, v+t \varphi_{2}\right) \in \Lambda .
$$

Clearly, we can take $\epsilon<\bar{\epsilon}$ satisfying

$$
h(t)\left(u+t \varphi_{1}, v+t \varphi_{2}\right) \in \Lambda^{+}, \quad \forall t<\epsilon,\left(\varphi_{1}, \varphi_{2}\right) \in E .
$$

Lemma 2.6. There exists a weak solution $\left(u_{0}, v_{0}\right)$ of the problem (1).

Proof: From Lemma 2.4 and Lemma 2.1, we have $\Lambda^{+} \neq \varnothing$ and $m=\inf _{(u, v) \in \Lambda} I(u, v)>-\infty$. By the Ekeland variational principle [14], there exists a minimizing sequence $\left\{\left(u_{k}, v_{k}\right)\right\} \subset \Lambda^{+}$which satisfies

$$
I\left(u_{k}, v_{k}\right)<m+\frac{1}{k}, \quad I\left(u_{k}, v_{k}\right) \leq I(u, v)+\frac{1}{k}\left\|\left(u-u_{k}, v-v_{k}\right)\right\|, \quad \forall(u, v) \in \Lambda^{+} .
$$

Since $I(u, v)=I(|u|,|v|)$, we assume $u_{k}, v_{k} \geq 0$ in $\Omega$ and there is a subsequence which is still denoted by $\left\{\left(u_{k}, v_{k}\right)\right\}$ and $u_{0}, v_{0} \geq 0$ such that

$$
\begin{cases}u_{k} \rightarrow u_{0}, \quad v_{k} \rightarrow v_{0}, & \text { weakly in } W_{0}^{1,2}(\Omega), \\ u_{k} \rightarrow u_{0}, \quad v_{k} \rightarrow v_{0}, & \text { strongly in } L^{p}(\Omega), p \in\left[1,2^{*}\right), \\ u_{k}(x) \rightarrow u_{0}(x), \quad v_{k}(x) \rightarrow v_{0}(x), & \text { a.e. in } \Omega .\end{cases}
$$

From Hardy inequality and Sobolev embedding theorem, it follows that

$$
\begin{cases}u_{k}^{2^{*}-1} \rightarrow u_{0}^{2^{*}-1}, \quad v_{k}^{2^{*}-1} \rightarrow v_{0}^{2^{*}-1}, & \text { weakly in } L^{\frac{2^{*}}{2^{*}-1}}(\Omega), \\ \frac{u_{k}}{|x|} \rightarrow \frac{u_{0}}{|x|}, \frac{v_{k}}{|x|} \rightarrow \frac{v_{0}}{|x|}, & \text { weakly in } L^{2} .\end{cases}
$$

By applying Lemma 2.5 , there exists a differentiable function $h_{k}(t)$ which satisfies

$$
h_{k}(0)=1, \quad h_{k}(t)\left(u_{k}+t \varphi_{1}, v_{k}+t \varphi_{2}\right) \in \Lambda^{+},
$$

where $\varphi_{1}, \varphi_{2} \in W_{0}^{1,2}(\Omega), t>0$ small enough. For the sake of simplicity, we write

$$
\begin{aligned}
& A(u, v)=\nabla u \nabla \varphi_{1}+\nabla v \nabla \varphi_{2}-\mu \frac{u \varphi_{1}}{|x|^{2}}-\mu \frac{v \varphi_{2}}{|x|^{2}}, \\
& B_{i}(u, v)=\alpha_{i} u^{\alpha_{i}-1} v^{\beta_{i}} \varphi_{1}+\beta_{i} u^{\alpha_{i}} v^{\beta_{i}-1} \varphi_{2}, i=1,2 .
\end{aligned}
$$

From the definition of $\Lambda$, we have

$$
\left\|\left(u_{k}, v_{k}\right)\right\|^{2}-\lambda \int_{\Omega}\left|u_{k}\right|^{\alpha_{1}}\left|v_{k}\right|^{\beta_{1}} \mathrm{~d} x-\int_{\Omega} f(x)\left|u_{k}\right|^{\alpha_{2}}\left|v_{k}\right|^{\beta_{2}} \mathrm{~d} x=0 .
$$

By applying (9), we deduce that 
On Positive Solution for a Class of Singular Elliptic System involving Critical Coupling Terms

$$
\begin{aligned}
& \frac{1}{k}\left[\left|h_{k}(t)-1\right|\left\|\left(u_{k}, v_{k}\right)\right\|+t h_{k}(t)\left\|\left(\varphi_{1}, \varphi_{2}\right)\right\|\right] \geq \frac{1}{k}\left\|h_{k}(t)\left(u_{k}+t \varphi_{1}, v_{k}+t \varphi_{2}\right)-\left(u_{k}, v_{k}\right)\right\| \\
\geq & I\left(u_{k}, v_{k}\right)-I\left(h_{k}(t)\left(u_{k}+t \varphi_{1}\right), h_{k}(t)\left(v_{k}+t \varphi_{2}\right)\right) \\
= & \frac{1}{2}\left\|\left(u_{k}, v_{k}\right)\right\|^{2}-\frac{\lambda}{2^{*}} \int_{\Omega}\left|u_{k}\right|^{\alpha_{1}}\left|v_{k}\right|^{\beta_{1}} \mathrm{~d} x-\frac{1}{q} \int_{\Omega} f(x)\left|u_{k}\right|^{\alpha_{2}}\left|v_{k}\right|^{\beta_{2}} \mathrm{~d} x \\
- & {\left[\frac{h_{k}^{2}(t)}{2}\left\|\left(u_{k}+t \varphi_{1}, v_{k}+t \varphi_{2}\right)\right\|^{2}-\frac{\lambda}{2^{*}} h_{k}^{2^{*}}(t) \int_{\Omega}\left|u_{k}+t \varphi_{1}\right|^{\alpha_{1}}\left|v_{k}+t \varphi_{2}\right|^{\beta_{1}} \mathrm{~d} x\right.} \\
- & \left.\frac{h_{k}^{q}(t)}{q} \int_{\Omega} f(x)\left|u_{k}+t \varphi_{1}\right|^{\alpha_{2}}\left|v_{k}+t \varphi_{2}\right|^{\beta_{2}} \mathrm{~d} x\right] \\
= & \frac{1-h_{k}^{2}(t)}{2}\left\|\left(u_{k}, v_{k}\right)\right\|^{2}-\lambda \frac{1-h_{k}^{2^{*}}(t)}{2^{*}} \int_{\Omega}\left|u_{k}\right|^{\alpha_{1}}\left|v_{k}\right|^{\beta_{1}} \mathrm{~d} x-\frac{1-h_{k}^{q}(t)}{q} \int_{\Omega} f(x)\left|u_{k}\right|^{\alpha_{2}}\left|v_{k}\right|^{\beta_{2}} \mathrm{~d} x \\
+ & \frac{h_{k}^{2}(t)}{2}\left(\left\|\left(u_{k}, v_{k}\right)\right\|^{2}-\left\|\left(u_{k}+t \varphi_{1}, v_{k}+t \varphi_{2}\right)\right\|^{2}\right) \\
- & \frac{\lambda h_{k}^{2^{*}}(t)}{2^{*}} \int_{\Omega}\left|u_{k}\right|^{\alpha_{1}}\left|v_{k}\right|^{\beta_{1}}-\left|u_{k}+t \varphi_{1}\right|^{\alpha_{1}}\left|v_{k}+t \varphi_{2}\right|^{\beta_{1}} \mathrm{~d} x \\
- & \frac{h_{k}^{q}(t)}{q} \int_{\Omega} f(x)\left(\left|u_{k}\right|^{\alpha_{2}}\left|v_{k}\right|^{\beta_{2}}-\left|u_{k}+t \varphi_{1}\right|^{\alpha_{2}}\left|v_{k}+t \varphi_{2}\right|^{\beta_{2}}\right) \mathrm{d} x .
\end{aligned}
$$

From Lemma 2.2 we know $u_{k}, v_{k} \neq 0$. Dividing the above inequality by $t>0$ and letting $t \rightarrow 0^{+}$, by (10) we find,

$$
\begin{aligned}
& \frac{1}{k}\left[\left|h_{k}^{\prime}(0)\right|\left\|\left(u_{k}, v_{k}\right)\right\|+\left\|\left(\varphi_{1}, \varphi_{2}\right)\right\|\right] \\
\geq & -\int_{\Omega} A\left(u_{k}, v_{k}\right) \mathrm{d} x+\lambda \int_{\Omega} B_{1}\left(u_{k}, v_{k}\right) \mathrm{d} x+\int_{\Omega} f(x) B_{2}\left(u_{k}, v_{k}\right) \mathrm{d} x .
\end{aligned}
$$

Namely,

$$
\begin{aligned}
& \int_{\Omega} f(x) B_{2}\left(u_{k}, v_{k}\right) \mathrm{d} x \\
\leq & \frac{1}{k}\left[\mid h_{k^{\prime}}(0)\left\|\left(u_{k}, v_{k}\right)\right\|+\left\|\left(\varphi_{1}, \varphi_{2}\right)\right\|\right]+\int_{\Omega} A\left(u_{k}, v_{k}\right) \mathrm{d} x-\lambda \int_{\Omega} B_{1}\left(u_{k}, v_{k}\right) \mathrm{d} x .
\end{aligned}
$$

From [12], there exists a constant $C_{2}>0$ such that $\left|h_{k}{ }^{\prime}(0)\right| \leq C_{2}$. Let $k \rightarrow \infty$ and by the Fatou Lemma, we have

$$
\int_{\Omega} f(x) B_{2}\left(u_{0}, v_{0}\right) \mathrm{d} x \leq \liminf _{k \rightarrow \infty} \int_{\Omega} f(x) B_{2}\left(u_{k}, v_{k}\right) \mathrm{d} x \leq \int_{\Omega} A\left(u_{0}, v_{0}\right) \mathrm{d} x-\lambda \int_{\Omega} B_{1}\left(u_{0}, v_{0}\right) \mathrm{d} x .
$$

Since $\left(\varphi_{1}, \varphi_{2}\right)$ is arbitrary, holds

$$
\int_{\Omega} A\left(u_{0}, v_{0}\right) \mathrm{d} x-\lambda \int_{\Omega} B_{1}\left(u_{0}, v_{0}\right) \mathrm{d} x-\int_{\Omega} f(x) B_{2}\left(u_{0}, v_{0}\right) \mathrm{d} x=0,
$$


Jie Yang and Zhi-Ying Deng

thus $\left(u_{0}, v_{0}\right)$ is a weak solution of problem (1).

Lemma 2.7. For any $\lambda>0,\left(u_{0}, v_{0}\right) \in \Lambda^{+}$.

Proof: Since $\left\{\left(u_{k}, v_{k}\right)\right\}$ is bounded in $W_{0}^{1,2}(\Omega)$, by Sobolev embedding theorem, there exists $C_{3}>0$ such that $\left|u_{k}\right|_{2^{2}}^{\alpha_{2}}\left|v_{k}\right|_{2^{*}}^{\beta_{2}} \leq C_{3}$. By the absolute continuity of integral, for all $\epsilon>0$ there exists $\delta>0$ such that if $E \subset \Omega$ and $|E|<\delta$, holds

$$
\int_{E} f^{\frac{2^{*}}{2^{*}-q}} \mathrm{~d} x<\epsilon^{\frac{2^{*}}{2^{*}-q}} .
$$

By the Holder inequality, it follows that:

$$
\begin{aligned}
\int_{E} f(x) u_{k}^{\alpha_{2}} v_{k}^{\beta_{2}} \mathrm{~d} x & \leq\left(\int_{E} u_{k}^{2^{*}} \mathrm{~d} x\right)^{\frac{\alpha_{2}}{2^{*}}}\left(\int_{E} v_{k}^{2^{*}} \mathrm{~d} x\right)^{\frac{\beta_{2}}{2^{*}}}\left(\int_{E} f^{\frac{2^{*}}{2^{*}-q}} \mathrm{~d} x\right)^{\frac{2^{*}-q}{2^{*}}} \\
& \leq\left|u_{k}\right|_{2^{*}}^{\alpha_{2}}\left|v_{k}\right|_{2^{*}}^{\beta_{2}}\left(\int_{E} f^{\frac{2^{*}}{2^{*}-q}} \mathrm{~d} x\right)^{\frac{2^{*}-q}{2^{*}}}<C_{3} \epsilon .
\end{aligned}
$$

Therefore, $\left\{\int_{\Omega} f(x)\left|u_{k}\right|^{1-s} \mathrm{~d} x \mid k=1,2, \cdots\right\}$ is equi-absolutely continuous. By applying the Vitali theorem [15], we have

$$
\lim _{k \rightarrow \infty} \int_{\Omega} f(x) u_{k}^{\alpha_{2}} v_{k}^{\beta_{2}} \mathrm{~d} x=\int_{\Omega} f(x) u_{0}^{\alpha_{2}} v_{0}^{\beta_{2}} \mathrm{~d} x .
$$

Taking $\phi_{1}=u_{0}, \phi_{2}=v_{0}$ in (11), we know that $\left(u_{0}, v_{0}\right) \in \Lambda$. Then, by the weak lower semi continuity of norm and (12), we have

$$
\begin{aligned}
& \inf _{(u, v) \in \Lambda^{+}} I(u, v)=\lim _{k \rightarrow \infty} I\left(u_{k}, v_{k}\right) \\
= & \lim _{k \rightarrow \infty}\left[\left(\frac{1}{2}-\frac{1}{2^{*}}\right)\left\|\left(u_{k}, v_{k}\right)\right\|^{2}-\left(\frac{1}{q}-\frac{1}{2^{*}}\right) \int_{\Omega} f(x)\left|u_{k}\right|^{\alpha_{2}}\left|v_{k}\right|^{\beta_{2}} \mathrm{~d} x\right] \\
\geq & \left(\frac{1}{2}-\frac{1}{2^{*}}\right)\left\|\left(u_{0}, v_{0}\right)\right\|^{2}-\left(\frac{1}{q}-\frac{1}{2^{*}}\right) \int_{\Omega} f(x)\left|u_{0}\right|^{\alpha_{2}}\left|v_{0}\right|^{\beta_{2}} \mathrm{~d} x \\
= & I\left(u_{0}, v_{0}\right) \geq \inf _{(u, v) \in \Lambda} I(u, v) .
\end{aligned}
$$

It is easy to know $\inf _{(u, v) \in \Lambda^{+}} I(u, v)=\inf _{(u, v) \in \Lambda} I(u, v)$ from Lemma 2.2 and Lemma 2.3. Therefore, the inequality signs in (13) should be equal signs. We can deduce that $\lim _{k \rightarrow \infty}\left\|\left(u_{k}, v_{k}\right)\right\|=\left\|\left(u_{0}, v_{0}\right)\right\|$. Namely, $u_{k} \rightarrow u_{0}, v_{k} \rightarrow v_{0}$ in $W_{0}^{1,2}(\Omega)$. From Lemma 2.4, we know $\Lambda^{+}$is closed, thus $\left(u_{0}, v_{0}\right) \in \Lambda^{+}$. 
On Positive Solution for a Class of Singular Elliptic System involving Critical Coupling Terms

\section{The proof of Theorem 1.1}

Let $\lambda^{*}=\min \left\{\lambda_{1}, \lambda_{2}, \lambda_{3}\right\}$, then Lemmas 2.1-2.7 hold for all $\lambda<\lambda^{*}$. Then we have $\left(u_{0}, v_{0}\right) \in \Lambda^{+}$is a weak solution of problem (1) and $I\left(u_{0}, v_{0}\right)=\inf _{(u, v) \in \Lambda} I(u, v)$ which means $\left(u_{0}, v_{0}\right)$ is a ground state solution.

Now we prove $u_{0}, v_{0}>0$ in $\Omega$. Since $u_{0}, v_{0} \geq 0$, for any $\varphi_{1}, \varphi_{2} \in W_{0}^{1,2}(\Omega)$, $\varphi_{1}, \varphi_{2} \geq 0, t>0$, we have

$$
\begin{aligned}
0 \leq & I\left(u_{0}+t \varphi_{1}, v_{0}+t \varphi_{2}\right)-I\left(u_{0}, v_{0}\right) \\
= & \frac{1}{2} \int_{\Omega}\left(\left|\nabla u_{0}+t \nabla \varphi_{1}\right|^{2}+\left|\nabla v_{0}+t \nabla \varphi_{2}\right|^{2}-\mu \frac{\left|u_{0}+t \varphi_{1}\right|^{2}}{|x|^{2}}-\mu \frac{\left|v_{0}+t \varphi_{2}\right|^{2}}{|x|^{2}}\right) \mathrm{d} x \\
& -\frac{\lambda}{2^{*}} \int_{\Omega}\left|u_{0}+t \varphi_{1}\right|^{\alpha_{1}}\left|v_{0}+t \varphi_{2}\right|^{\beta_{1}} \mathrm{~d} x-\frac{1}{2} \int_{\Omega}\left(\left|\nabla u_{0}\right|^{2}+\left|\nabla v_{0}\right|^{2}-\mu \frac{\left|u_{0}\right|^{2}}{|x|^{2}}-\mu \frac{\left|v_{0}\right|^{2}}{|x|^{2}}\right) \mathrm{d} x \\
& -\frac{1}{q} \int_{\Omega} f(x)\left|u_{0}+t \varphi_{1}\right|^{\alpha_{2}}\left|v_{0}+t \varphi_{2}\right|^{\beta_{2}} \mathrm{~d} x+\frac{\lambda}{2^{*}} \int_{\Omega}\left|u_{0}\right|^{\alpha_{1}}\left|v_{0}\right|^{\beta_{1}} \mathrm{~d} x \\
& +\frac{1}{q} \int_{\Omega} f(x)\left|u_{0}\right|^{\alpha_{2}}\left|v_{0}\right|^{\beta_{2}} \mathrm{~d} x \\
\leq & \frac{1}{2} \int_{\Omega}\left(\left|\nabla u_{0}+t \nabla \varphi_{1}\right|^{2}+\left|\nabla v_{0}+t \nabla \varphi_{2}\right|^{2}-\mu \frac{\left|u_{0}+t \varphi_{1}\right|^{2}}{|x|^{2}}-\mu \frac{\left|v_{0}+t \varphi_{2}\right|^{2}}{|x|^{2}}\right) \mathrm{d} x \\
& -\frac{1}{2} \int_{\Omega}\left(\left|\nabla u_{0}\right|^{2}+\left|\nabla v_{0}\right|^{2}-\mu \frac{\left|u_{0}\right|^{2}}{|x|^{2}}-\mu \frac{\left|v_{0}\right|^{2}}{|x|^{2}}\right) \mathrm{d} x .
\end{aligned}
$$

This implies

$$
\begin{aligned}
& \int_{\Omega}\left(\left|\nabla u_{0}+t \nabla \varphi_{1}\right|^{2}+\left|\nabla v_{0}+t \nabla \varphi_{2}\right|^{2}-\mu \frac{\left|u_{0}+t \varphi_{1}\right|^{2}}{|x|^{2}}-\mu \frac{\left|v_{0}+t \varphi_{2}\right|^{2}}{|x|^{2}}\right) \mathrm{d} x \\
& -\int_{\Omega}\left(\left|\nabla u_{0}\right|^{2}+\left|\nabla v_{0}\right|^{2}-\mu \frac{\left|u_{0}\right|^{2}}{|x|^{2}}-\mu \frac{\left|v_{0}\right|^{2}}{|x|^{2}}\right) \mathrm{d} x \geq 0,
\end{aligned}
$$

dividing by $t>0$ and letting $t \rightarrow 0$, we get

$$
\int_{\Omega}\left(\nabla u_{0} \nabla \varphi_{1}+\nabla v_{0} \nabla \varphi_{2}-\mu \frac{u_{0} \varphi_{1}+v_{0} \varphi_{2}}{|x|^{2}}\right) \mathrm{d} x \geq 0
$$

Then, by the strong maximum principle, we have $u_{0}, v_{0}>0$ in $\Omega$. This completes the proof of Theorem 1.1.

Acknowledgements. This work is supported by the National Natural Science Foundation of China (grant Nos. 11971339; 11601052) and funded by Chongqing Research Program of Basic Research and Frontier Technology (grant No. cstc2017jcyjBX0037). 


\section{Jie Yang and Zhi-Ying Deng}

\section{REFERENCES}

1. X.Wang, P.Zhao and L.Zhang, The existence and multiplicity of classical positive solutions for a singular nonlinear elliptic problem with any growth exponents, Nonlinear Analysis: Theory, Methods and Applications, 101 (2014) 37-46.

2. Y.Sang, X.Luo and Z.Zhu, Two positive solutions for quasilinear elliptic equations with singularity and critical exponents, Boundary Value Problems, 1 (2018) 1-19.

3. N.Hirano, C.Saccon and N.Shioji, Existence of multiple positive solutions for singular elliptic problems with concave and convex nonlinearities, Advances in Differential Equations, 9(1-2) (2004) 197-220.

4. J.Zhang and Z.Wei, Existence of multiple positive solutions to singular elliptic systems involving critical exponents, Nonlinear Analysis Theory, Methods and Applications, 75(2) (2012) 559-573.

5. D.Kang and L.Xu, A critical surface for the solutions to singular elliptic systems, Journal of Mathematical Analysis and Applications, 472(2) (2019) 2017-2033.

6. D.Kang, J.Gong and X.Duan, Existence of solutions for two singular critical elliptic systems, Journal of South-Central University for Nationalities, 35(4) (2016) 126131.

7. W.Zhang, The existence of positive ground state solutions for a singular quasilinear elliptic systems involving critical exponents, Mathematics in practice and theory, 44(21) (2014) 286-295.

8. T.S.Hsu, H.L.Li, Multiplicity of positive solutions for singular elliptic systems with critical Sobolev-Hardy and concave exponents, Acta Mathematica Scientia, 31B(3) (2011) 791-804.

9. A.T.Duong, D.Lan, P.Q.Le, et al, On the nonexistence of stable solutions of subelliptic systems with negative exponents, Complex Variables, Theory and Application: An International Journal, 64(12) (2019) 2117-2129.

10. M.Ghergu, On a class of singular elliptic systems, Nonlinear Analysis: Theory, Methods and Applications, 119 (2015) 98-105.

11. Z.Zhang, Positive solutions of Lane-Emden systems with negative exponents: Existence, boundary behavior and uniqueness, Fuel and Energy Abstracts, 74(16) (2011) 5544-5553.

12. Y.Sun, S.Wu, Y.Long, Combined effects of singular and superlinear nonlinearities in some singular boundary value problems, Journal of Differential Equations, 176(2) (2001) 511-531.

13. G.Tarantello, On nonhomogeneous elliptic equations involving critical Sobolev exponent, Non Linear Analysis. 9(3) (1992) 281-304.

14. M.Struwe, Variational Methods, Springer-Verlag, New York, Berlin, 1990.

15. W.Rudin, Real and complex analysis, McGraw-Hill, New York, London, 1966. 